\title{
A SHOCK LAYER ARISING AS THE SOURCE TERM COLLAPSES IN THE P(X)-LAPLACIAN EQUATION
}

\begin{abstract}
We study the Cauchy-Dirichlet problem for the $p(\boldsymbol{x})$-Laplacian equation with a regular finite nonlinear minor term. The minor term depends on a small parameter $\varepsilon>0$ and, as $\varepsilon \rightarrow 0$, converges weakly ${ }^{\star}$ to the expression incorporating the Dirac delta function, which models a shock (impulsive) loading. We establish that the shock layer, associated with the Dirac delta function, is formed as $\varepsilon \rightarrow 0$, and that the family of weak solutions of the original problem converges to a solution of a two-scale microscopicmacroscopic model. This model consists of two equations and the set of initial and boundary conditions, so that the 'outer' macroscopic solution beyond the shock layer is governed by the usual homogeneous $p(\boldsymbol{x})$-Laplacian equation, while the shock layer solution is defined on the microscopic level and obeys the ordinary differential equation derived from the microstructure of the shock layer profile.
\end{abstract}

Key words: parabolic equation, nonstandard growth, variable nonlinearity, non-instantaneous impulse, energy solution, shock layer

2010 Mathematical Subject Classification: 35K67, 35B65, $35 K 55,35 K 99$

1. Introduction. In this article, we study the Cauchy-Dirichlet problem for the isotropic $p(\boldsymbol{x})$-parabolic equation with a nonlinear minor (source) term:

$$
\left\{\begin{array}{l}
\partial_{t} u_{\varepsilon}=\operatorname{div}_{x}\left(\left|\nabla_{x} u_{\varepsilon}\right|^{p(\boldsymbol{x})-2} \nabla_{x} u_{\varepsilon}\right)+K_{\varepsilon}^{\tau}(t) \beta\left(\boldsymbol{x}, u_{\varepsilon}\right), \quad(\boldsymbol{x}, t) \in Q_{T} \\
u_{\varepsilon}=0 \text { on } \Gamma_{T}, \quad u_{\varepsilon}(\boldsymbol{x}, 0)=u_{0}(\boldsymbol{x}) \text { in } \Omega
\end{array}\right.
$$

In the formulation of problem (1), $\Omega \subset \mathbb{R}^{d}$ is a bounded simpleconnected domain with a $C^{1}$-boundary $\partial \Omega, T \in(0,+\infty)$ is an arbitrarily

(C) Petrozavodsk State University, 2020 
fixed time. We denote $Q_{T}:=\Omega \times(0, T)$ and $\Gamma_{T}:=\partial \Omega \times(0, T)$, which are the space-time cylinder and its lateral boundary, respectively. By $\tau$ we denote the fixed time strictly between 0 and $T$, i. e., $\tau \in(0, T)$.

The function $u_{\varepsilon}=u_{\varepsilon}(\boldsymbol{x}, t)$ is a sought one. The exponent $p=p(\boldsymbol{x})$ is given. The kernel $K_{\varepsilon}^{\tau}=K_{\varepsilon}^{\tau}(t)$ is supported on $[\tau-\varepsilon, \tau]$ and defined by the formula

$$
K_{\varepsilon}^{\tau}(t)=\frac{2}{\varepsilon} K\left(\frac{t-\tau}{\varepsilon}\right) \theta(\tau-t), \quad t \in(0, T),
$$

where $K=K(\vartheta)$ is an even nonnegative smooth function supported on the segment $\{-1 \leqslant \vartheta \leqslant 1\}$, with the mean value equal to unity, i. e., $\int_{-1}^{1} K(\vartheta) d \vartheta=1 ; \theta(\vartheta)=\mathbf{1}_{(\vartheta \geqslant 0)}$ is the Heaviside step function, and $\varepsilon>0$ is a small parameter. The source function $\beta=\beta(\boldsymbol{x}, z)$ is smooth and satisfies the growth condition

$$
\begin{aligned}
& |\beta(\boldsymbol{x}, z)| \leqslant \beta_{0}|z|^{\lambda-1}+\beta_{1}(\boldsymbol{x}), \quad \text { where } \\
& \lambda \in(1,2], \quad \beta_{0}=\mathrm{const}>0, \quad \beta_{1} \in L^{\lambda^{\prime}}(\Omega), \quad \frac{1}{\lambda}+\frac{1}{\lambda^{\prime}}=1,
\end{aligned}
$$

and the finiteness condition

$$
\beta(\boldsymbol{x}, z)=0 \text { for }(\boldsymbol{x}, z) \in \partial \Omega \times \mathbb{R} .
$$

The structure of function $K_{\varepsilon}^{\tau}$ yields that it approximates the left-sided Dirac delta function in the sense that

$$
K_{\varepsilon}^{\tau}(t) \underset{\varepsilon \rightarrow 0+}{\longrightarrow} \delta_{(t=\tau-0)} \text { weakly }{ }^{\star} \text { in } \mathcal{M}(0, T) .
$$

Furthermore, $\varepsilon K_{\varepsilon}^{\tau}(\tau+\varepsilon \vartheta)=\left\{\begin{array}{ll}2 K(\vartheta) & \text { if } \quad \vartheta \leqslant 0, \\ 0 & \text { if } \vartheta>0,\end{array}\right.$ and

$$
\int_{0}^{t} K_{\varepsilon}^{\tau}(s) d s \leqslant 1, \quad \int_{0}^{T} K_{\varepsilon}^{\tau}(s) d s=1 .
$$

Note that $K$ may have the form of the classical Friedrichs mollifier.

With respect to the exponent $p$, we assume that it takes values in the interval $\left(p^{-}, p^{+}\right) \subset(1,+\infty)$ and is log-continuous, i. e.,

$$
\left.\left|p\left(\boldsymbol{x}_{2}\right)-p\left(\boldsymbol{x}_{1}\right)\right| \leqslant \omega\left(\left|\boldsymbol{x}_{2}-\boldsymbol{x}_{1}\right|\right) \quad \text { (for small }\left|\boldsymbol{x}_{2}-\boldsymbol{x}_{1}\right|\right),
$$


with the modulus of continuity satisfying the condition

$$
\limsup _{s \rightarrow 0+} \omega(s) \ln \frac{1}{s}=C<\infty, \quad C=\text { const. }
$$

Initial function $u_{0}$ is given.

We are interested in the energy solutions of problem (1). Let

$$
\begin{aligned}
\mathbf{W}\left(Q_{T}\right) & =\left\{u: Q_{T} \mapsto \mathbb{R}:\left.u\right|_{\Gamma_{T}}=0, u \in L^{2}\left(Q_{T}\right),\left|\nabla_{x} u\right|^{p(\boldsymbol{x})} \in L^{1}\left(Q_{T}\right)\right\} \\
\mathbf{Z} & =\left\{\zeta=\zeta(\boldsymbol{x}, t): \zeta \in \mathbf{W}\left(Q_{T}\right), \partial_{t} \zeta \in \mathbf{W}^{\prime}\left(Q_{T}\right)\right\}
\end{aligned}
$$

$\mathbf{W}^{\prime}\left(Q_{T}\right)$ be the dual of $\mathbf{W}\left(Q_{T}\right)$, and $\langle\cdot, \cdot\rangle_{\mathbf{W}^{\prime}\left(Q_{T}\right), \mathbf{W}\left(Q_{T}\right)}$ be the duality bracket between $\mathbf{W}\left(Q_{T}\right)$ and $\mathbf{W}^{\prime}\left(Q_{T}\right)$. Both $\mathbf{W}\left(Q_{T}\right)$ and $\mathbf{Z}$ are Banach spaces; their basic properties can be found in [5, Ch. 1].

Now we are in a position to formulate the following notion.

Definition 1. (Energy solution.) A function $u_{\varepsilon}: Q_{T} \mapsto \mathbb{R}$ is called a weak (energy) solution of problem (1) if

1) $u_{\varepsilon} \in \mathbf{W}\left(Q_{T}\right), \partial_{t} u_{\varepsilon} \in \mathbf{W}^{\prime}\left(Q_{T}\right)$;

2) the integral equality

$$
\begin{aligned}
& \left\langle\partial_{t} u_{\varepsilon}, \zeta\right\rangle_{\mathbf{W}^{\prime}\left(Q_{T}\right), \mathbf{W}\left(Q_{T}\right)}+ \\
& \quad+\int_{Q_{T}}\left(\left|\nabla_{x} u_{\varepsilon}\right|^{p(\boldsymbol{x})-2} \nabla_{x} u_{\varepsilon} \cdot \nabla_{x} \zeta-K_{\varepsilon}^{\tau}(t) \beta\left(\boldsymbol{x}, u_{\varepsilon}\right) \zeta\right) d \boldsymbol{x} d t=0
\end{aligned}
$$

holds for every test function $\zeta \in \mathbf{Z}$;

3) the limiting relation $\int_{\Omega}\left(u_{\varepsilon}(\boldsymbol{x}, t)-u_{0}(\boldsymbol{x})\right) \eta(\boldsymbol{x}) d \boldsymbol{x} \rightarrow 0$, as $t \rightarrow 0+$, holds for every test function $\eta \in C_{0}^{\infty}(\Omega)$.

Remark 1. According to the well-known embedding theorem [16, Ch. 3, Lemma 1.2], if $u_{\varepsilon}$ is an energy solution of the problem (1) and $\zeta$ belongs to $\mathbf{Z}$, then $u_{\varepsilon}$ and $\zeta$ belong to $C\left([0, T] ; L^{2}(\Omega)\right)$.

In line with a widely accepted terminology [1], [18], equation $(1)_{1}$ can be referred to as a non-instantaneous impulsive differential equation due to the presence of the term $K_{\varepsilon}^{\tau}(t) \beta\left(\boldsymbol{x}, u_{\varepsilon}\right)$ that approximates the Dirac deltatype source $\delta_{(t=\tau)} \beta(\boldsymbol{x}, u)$. Also, equations of the type $(1)_{1}$ fall into the class 
of equations with nonstandard growth, or variable nonlinearity. Equation $(1)_{1}$ degenerates or becomes singular at the points where $\left|\nabla_{x} u\right|^{p(\boldsymbol{x})-2}$ vanishes or becomes infinite; in this work, both degenerate and singular cases are allowed. There already exists a wide literature on the issues of existence and uniqueness of energy solutions of the problem (1) understood in the sense of Definition 1 for a fixed $\varepsilon>0$. For further information on this topic, see [2-5], [7] and references therein.

In this article, the statement of existence of an energy solution to problem (1) for every fixed $\varepsilon \in\left(0, \varepsilon_{0}\right]$ (with $\varepsilon_{0}$ small enough) is due to the already established theory, and we focus on constructing the estimates, uniform in $\varepsilon$. After this, we pass to limit in the problem (1) as $\varepsilon \rightarrow 0$. Before turning directly to formulation of the main results and to their proofs, let us pay attention to one subtle point in the study, which can be misleading if one neglects the mathematical rigor.

Notice that the straightforward substitution of $\delta_{(t=\tau-0)}=w^{\star}-\lim _{\varepsilon \rightarrow 0} K_{\varepsilon}^{\tau}$ for $K_{\varepsilon}^{\tau}$ into (1) yields the impulsive $p(\boldsymbol{x})$-Laplacian equation

$$
\partial_{t} u=\operatorname{div}_{x}\left(\left|\nabla_{x} u\right|^{p(\boldsymbol{x})-2} \nabla_{x} u\right)+\delta_{(t=\tau-0)}(t) \beta(\boldsymbol{x}, u), \quad(\boldsymbol{x}, t) \in Q_{T},
$$

which is equivalent in the sense of distributions to the system consisting of the homogeneous $p(\boldsymbol{x})$-Laplacian equation (see (14a)) and the standard impulsive condition

$$
u(\boldsymbol{x}, \tau+0)=u(\boldsymbol{x}, \tau-0)+\beta(\boldsymbol{x}, u(\boldsymbol{x}, \tau-0)) .
$$

At first glance, (7) looks like the proper limiting equation for $(1)_{1}$ and, consequently, the system (14a), (8), and (1) looks like the proper limiting system for (1), as $\varepsilon \rightarrow 0+$. On the other hand, numerous observations in the theory of impulsive ordinary differential equations signal that the situation with the limit may turn to be drastically more sophisticated [6], [8], [11]. For example [6], consider the first-order ordinary differential equation

$$
\frac{d f_{\varepsilon}(x)}{d x}=\alpha f_{\varepsilon}(x) \delta_{\varepsilon}(x), \quad x \in \mathbb{R}
$$

with

$$
\alpha=\text { const, } \quad \delta_{\varepsilon}(x)=\left\{\begin{array}{lll}
\frac{1}{2 \varepsilon} & \text { if } \quad x \in(-\varepsilon, \varepsilon), \quad \varepsilon>0 . \\
0 & \text { if } \quad|x| \geqslant \varepsilon,
\end{array}\right.
$$

It is easy to deduce that the function $\delta_{\varepsilon}$ weakly ${ }^{\star}$ approximates the Dirac delta function $\delta_{(x=0)}$ concentrated at the origin $(x=0)$ and to calculate 
explicitly the solution of (9) for every fixed $\varepsilon>0$ :

$$
f_{\varepsilon}(x)=C \exp \left(\alpha \int_{0}^{x} \delta_{\varepsilon}(y) d y\right)= \begin{cases}C \exp \left(-\frac{\alpha}{2}\right) & \text { if } \quad x \leqslant-\varepsilon, \\ C \exp \left(\frac{\alpha x}{2 \varepsilon}\right) \quad \text { if } \quad-\varepsilon<x<\varepsilon, \\ C \exp \left(\frac{\alpha}{2}\right) \quad \text { if } \quad x \geqslant \varepsilon .\end{cases}
$$

It is easy to establish that the limiting (as $\varepsilon \rightarrow 0+$ ) function $f=\lim _{\varepsilon \rightarrow 0} f_{\varepsilon}$ meets the jump condition

$$
f(0+)-f(0-)=\tanh \left(\frac{\alpha}{2}\right)[f(0+)+f(0-)] .
$$

At the same time, the solution of equation $\frac{d f(x)}{d x}=\alpha f(x) \delta_{(x=0)}$ meets the standard impulsive condition

$$
f(0+)-f(0-)=\frac{\alpha}{2}[f(0+)+f(0-)],
$$

and we immediately notice the discrepancy between (10) and (11), since these two conditions coincide only for $\alpha=0$, i.e., in the trivial case.

Looking ahead, we note that in our article we encounter a somewhat similar situation, as in the above example. As a matter of fact, the guess that the system (14a), (8), and (1) 2 is the limiting form of the system (1) is wrong. In order to derive the correct limiting formulation, we thoroughly treat the system (1) as $\varepsilon \rightarrow 0+$ on the rigorous mathematical level further in Section 4. This study eventually brings us to the notion of a shock layer arising due to the collapsing term $K_{\varepsilon}^{\tau}(t) \beta\left(\boldsymbol{x}, u_{\varepsilon}\right)$.

Now let us turn to exposition of the main results and their proofs.

2. The main results. The following theorem states the existence of solutions and provides the uniform estimates in $\varepsilon$, which is the first main result of the article.

Theorem 1. Assume that $u_{0} \in L^{2}(\Omega)$ and functions $p, K_{\varepsilon}^{\tau}$, and $\beta$ satisfy the requirements stated in Introduction.

Then, for every $\varepsilon>0$ the problem (1) has at least one weak (energy) solution $u \in \mathbf{W}\left(Q_{T}\right)$ satisfying the energy estimate

$$
\begin{aligned}
\left\|u_{\varepsilon}\right\|_{L^{\infty}\left(0, T ; L^{2}(\Omega)\right)}^{2} & +\int_{Q_{T}}\left|\nabla_{x} u_{\varepsilon}\right|^{p(\boldsymbol{x})} d \boldsymbol{x} d t \leqslant \\
& \leqslant C\left(\left\|u_{0}\right\|_{L^{2}(\Omega)}^{2}+\left\|\beta_{1}\right\|_{L^{\lambda^{\prime}(\Omega)}}^{\lambda^{\prime}}+1\right),
\end{aligned}
$$


with a constant $C$ independent of $u_{\varepsilon}$ and $\varepsilon$.

Moreover, if $u_{0} \in L^{\infty}(\Omega)$ then $u_{\varepsilon} \in L^{\infty}\left(Q_{T}\right)$ and the maximum principle holds:

$$
\begin{aligned}
\sup _{t \in[0, T]}\left\|u_{\varepsilon}(\cdot, t)\right\|_{L^{\infty}(\Omega)} \leqslant e^{\beta_{0}}\left\|u_{0}\right\|_{L^{\infty}(\Omega)}+ \\
\quad+\left(e^{\beta_{0}}-1\right)\left(1+\frac{\left\|\beta_{1}\right\|_{L^{\infty}(\Omega)}}{\beta_{0}}\right)=: M_{(13)},
\end{aligned}
$$

where constant $M_{(13)}$ does not depend on $\varepsilon$.

The second main result of the article deals with the limiting transition in the problem (1) as $\varepsilon$ tends to zero. It reads as follows:

Theorem 2. Assume that $u_{0} \in L^{\infty}(\Omega)$, functions $p, K_{\varepsilon}^{\tau}$, and $\beta$ satisfy the requirements stated in Introduction, and $\left\{u_{\varepsilon}\right\}_{\varepsilon>0}$ is the family of weak (energy) solutions of problem (1) in the sense of Definition 1.

The following assertions hold true.

1) The family $\left\{u_{\varepsilon}\right\}_{\varepsilon>0}$ is relatively compact in $L^{q}\left(Q_{T}\right)$ for every fixed $q \in[1,+\infty)$. More precisely, there exists a subsequence from $\left\{u_{\varepsilon}\right\}_{\varepsilon>0}$ (still labelled by $\varepsilon$ ) and a limit function $u \in L^{\infty}\left(Q_{T}\right) \cap \mathbf{W}(\Omega \times(0, \tau)) \cap$ $\mathbf{W}(\Omega \times(\tau, T))$, such that $u_{\varepsilon} \underset{\varepsilon \rightarrow 0+}{\longrightarrow} u$ strongly in $L^{q}\left(Q_{T}\right)$.

2) The family of rescaled solutions $\left\{\bar{u}_{\varepsilon}: \Omega \times[-1,0] \mapsto \mathbb{R}\right\}$ defined by the formula $\bar{u}_{\varepsilon}(\boldsymbol{x}, \bar{t}) \stackrel{\text { def }}{=} u_{\varepsilon}(\boldsymbol{x}, \tau+\varepsilon \bar{t}), \bar{t} \in[-1,0], \varepsilon>0$, is relatively compact in $L^{q}(\Omega \times(-1,0))$ for every fixed $q \in[1,+\infty)$. More precisely, there exists a subsequence from $\left\{\bar{u}_{\varepsilon}\right\}_{\varepsilon>0}$ (still labelled by $\varepsilon$ ) and a limiting function $\bar{u} \in L^{\infty}(\Omega \times(-1,0))$, such that $\bar{u}_{\varepsilon} \underset{\varepsilon \rightarrow 0+}{\longrightarrow} \bar{u}$ strongly in $L^{q}(\Omega \times(-1,0))$.

3) The pair of functions $\{u, \bar{u}\}$, defined in assertions 1 and 2 of this theorem, is a generalized solution of the following impulsive-initialboundary-value problem:

$$
\begin{aligned}
& \partial_{t} u=\operatorname{div}_{x}\left(\left|\nabla_{x} u\right|^{p(\boldsymbol{x})-2} \nabla_{x} u\right),(\boldsymbol{x}, t) \in Q_{T} \backslash\{t=\tau\}, \\
& \partial_{\bar{t}} \bar{u}=2 K(\bar{t}) \beta(\boldsymbol{x}, \bar{u}),(\boldsymbol{x}, \bar{t}) \in \Omega \times(-1,0), \\
& u=0 \text { on } \Gamma_{T} \\
& u(\boldsymbol{x}, 0)=u_{0}(\boldsymbol{x}) \text { in } \Omega \\
& u(\boldsymbol{x}, \tau-0)=\bar{u}(\boldsymbol{x},-1+0), u(\boldsymbol{x}, \tau+0)=\bar{u}(\boldsymbol{x}, 0-) \text { in } \Omega .
\end{aligned}
$$


4) The problem (14) has at most one generalized solution. In other words, the generalized solution $\{u, \bar{u}\}$, defined in assertions 1) and 2) of this theorem, is unique.

In the formulation of problem (14), equation (14a) is understood in the weak sense, equation (14b) holds a. e. in $\Omega \times(-1,0)$, the homogeneous boundary condition (14c), the initial condition (14d), and the matching conditions (14e) are understood in the sense of strong traces in $L^{1}$.

We call the set $\Omega \times\{-1<\bar{t}<0\}$ the shock layer following the classical terminology accepted in fluid dynamics, see, e.g., [15, §57]. Notice that $(14 \mathrm{~b})$ is an ordinary differential equation for the sought function $\bar{u}$ on $\Omega \times\{-1<\bar{t}<0\}$, where $\bar{t}$ is 'the fast' (microscopic) time variable and the spatial coordinate $\boldsymbol{x}$ plays the role of a parameter, while (14a) is the homogeneous $p(\boldsymbol{x})$-Laplacian equation for the sought 'outer' solution $u$ beyond the shock layer.

Theorems 1 and 2 are proved further in Sections 3 and 4, respectively.

\section{Proof of Theorem 1.}

3.1. Existence of weak (energy) solutions. Existence of an energy solution $u_{\varepsilon}$ to the problem (1) for every fixed $\varepsilon>0$ directly follows from the results [5, Section 4.2, Theorem 4.2; Section 4.9, Remark on p. 172]. In the singular case $p(\boldsymbol{x}) \leqslant p^{+}<2$ we can also refer to [4, Theorems 1.1 and 1.3], where existence of more regular, namely, strong solutions is established.

3.2. Maximum principle uniform in $\varepsilon$. Let us introduce $Y_{2 k}(t):=\left\|u_{\varepsilon}(\cdot, t)\right\|_{L^{2 k}(\Omega)}$. For every $k$ the function $\left|u_{\varepsilon}\right|^{2 k-2} u_{\varepsilon}$ can be taken as a test function in (6). Using (2) and the formula of integration by parts [5, Section 1.5, Lemma 1.20], we arrive at the energy relation

$$
\frac{1}{2 k} \frac{d Y_{2 k}^{2 k}(t)}{d t}+(2 k-1) \int_{\Omega}\left|u_{\varepsilon}(\boldsymbol{x}, t)\right|^{2 k-2}\left|\nabla_{x} u_{\varepsilon}(\boldsymbol{x}, t)\right|^{p(\boldsymbol{x})} d \boldsymbol{x}=I(t), t<T,
$$

where we denote

$$
I(t):=K_{\varepsilon}^{\tau}(t) \int_{\Omega}\left|u_{\varepsilon}\right|^{2 k-2} u_{\varepsilon} \beta\left(\boldsymbol{x}, u_{\varepsilon}\right) d \boldsymbol{x} .
$$

Using the growth condition (3) and further applying the Hölder inequality, 
we get the estimate

$$
\begin{aligned}
|I(t)| & \leqslant K_{\varepsilon}^{\tau}(t) \int_{\Omega}\left|u_{\varepsilon}\right|^{2 k-1}\left(\beta_{0}\left|u_{\varepsilon}\right|^{\lambda-1}+\beta_{1}(\boldsymbol{x})\right) d \boldsymbol{x} \leqslant \\
& \leqslant K_{\varepsilon}^{\tau}(t)\left(\beta_{0} Y_{2 k}^{2 k-2+\lambda}(t)|\Omega|^{\frac{2-\lambda}{2 k}}+Y_{2 k}^{2 k-1}(t)\left\|\beta_{1}\right\|_{L^{2 k}(\Omega)}\right) .
\end{aligned}
$$

By combining this estimate with (15) followed by discarding the second term in the left-hand side, we get

$$
\begin{aligned}
Y_{2 k}^{2 k-1} \frac{d Y_{2 k}}{d t}(t) & \leqslant|I(t)| \leqslant \\
& \leqslant K_{\varepsilon}^{\tau}(t)\left(\beta_{0} Y_{2 k}^{2 k-2+\lambda}(t)|\Omega|^{\frac{2-\lambda}{2 k}}+Y_{2 k}^{2 k-1}(t)\left\|\beta_{1}\right\|_{L^{2 k}(\Omega)}\right) .
\end{aligned}
$$

Dividing both parts by $Y_{2 k}^{2 k-1}$, we arrive at the differential inequality

$$
\begin{aligned}
\frac{d Y_{2 k}}{d t}(t) & \leqslant K_{\varepsilon}^{\tau}(t)\left(\beta_{0}|\Omega|^{\frac{2-\lambda}{2 k}} Y_{2 k}^{\lambda-1}(t)+\left\|\beta_{1}\right\|_{L^{2 k}(\Omega)}\right) \leqslant \\
& \stackrel{\lambda \in(1,2]}{\leqslant} K_{\varepsilon}^{\tau}(t)\left(\beta_{0}|\Omega|^{\frac{2-\lambda}{2 k}}\left(Y_{2 k}(t)+1\right)+\left\|\beta_{1}\right\|_{L^{2 k}(\Omega)}\right) .
\end{aligned}
$$

Applying Grönwall's lemma and the bound (5), we arrive at the inequality

$$
Y_{2 k}(t) \leqslant e^{\beta_{0}|\Omega|^{\frac{2-\lambda}{2 k}}} Y_{2 k}(0)+\left(e^{\beta_{0}|\Omega|^{\frac{2-\lambda}{2 k}}}-1\right)\left(1+\frac{\left\|\beta_{1}\right\|_{L^{2 k}(\Omega)}}{\beta_{0}|\Omega|^{\frac{2-\lambda}{2 k}}}\right) .
$$

Passing to the limit as $k \rightarrow \infty$ and taking into account that $|\Omega|<\infty$ and $\lim _{k \rightarrow \infty}|\Omega|^{\frac{2-\lambda}{2 k}}=1$ for $\lambda \in(1,2]$, we obtain the desired bound (13).

3.3. Energy estimate for $\boldsymbol{\nabla}_{\boldsymbol{x}} \boldsymbol{u}_{\varepsilon}$. Joining (15) and (17) with $k=1$, we arrive at the inequality

$$
\begin{aligned}
\frac{1}{2} \frac{d}{d t} Y_{2}^{2}(t) & +\int_{\Omega}\left|\nabla_{x} u_{\varepsilon}(\boldsymbol{x}, t)\right|^{p(\boldsymbol{x})} d \boldsymbol{x} \leqslant \\
& \leqslant K_{\varepsilon}^{\tau}(t)\left(\left(\beta_{0}+\frac{1}{\lambda}\right)|\Omega|^{\frac{2-\lambda}{2}}\left(Y_{2}^{2}(t)+1\right)+\frac{1}{\lambda^{\prime}}\left\|\beta_{1}\right\|_{L^{\lambda^{\prime}}(\Omega)}^{\lambda^{\prime}}\right) .
\end{aligned}
$$

Arguing the same way as for (16), from (18) we obtain the inequalities

$$
\begin{aligned}
Y_{2}^{2}(t) & \leqslant e^{2\left(\beta_{0}+\frac{1}{\lambda}\right)|\Omega|^{\frac{2-\lambda}{2}}} Y_{2}^{2}(0)+ \\
& +\left(e^{2\left(\beta_{0}+\frac{1}{\lambda}\right)|\Omega|^{\frac{2-\lambda}{2}}}-1\right)\left(1+\frac{2\left\|\beta_{1}\right\|_{L^{\lambda^{\prime}}(\Omega)}^{\lambda^{\prime}}}{\lambda^{\prime}\left(\beta_{0}+\frac{1}{\lambda}\right)|\Omega|^{\frac{2-\lambda}{2}}}\right),
\end{aligned}
$$




$$
\begin{aligned}
& \int_{0}^{t} \int_{\Omega}\left|\nabla_{x} u_{\varepsilon}(\boldsymbol{x}, s)\right|^{p(\boldsymbol{x})} d \boldsymbol{x} d s \leqslant \\
& \quad \leqslant \frac{1}{2} Y_{2}^{2}(0)+\left(\beta_{0}+\frac{1}{\lambda}\right)|\Omega|^{\frac{2-\lambda}{2}}\left(\sup _{s \in[0, t]} Y_{2}^{2}(s)+1\right)+\frac{1}{\lambda^{\prime}}\left\|\beta_{1}\right\|_{L^{\lambda^{\prime}}(\Omega)}^{\lambda^{\prime}}
\end{aligned}
$$

this completes the proof of Theorem 1.

\section{Proof of Theorem 2.}

4.1. Relative compactness of $\left\{\mathbf{u}_{\varepsilon}\right\}_{\varepsilon>0}$. We start proving the theorem by finding the equi-continuity property of the family $\left\{u_{\varepsilon}\right\}_{\varepsilon>0}$. Namely, we establish the following lemma:

Lemma 1. For any $\varepsilon>0$, for every pair $(\varrho, h)$ of rather small positive parameters (i.e., $\varrho_{i}>0, h>0$ ), and for every $q \geqslant \max \left\{2, p^{-}\right\}$, the weak (energy) solution of the problem (1) satisfies the estimate

$$
\int_{0}^{T-h} \int_{\Omega_{\varrho}}\left|u_{\varepsilon}(\boldsymbol{x}+\boldsymbol{\varrho}, s+h)-u_{\varepsilon}(\boldsymbol{x}, s)\right|^{q} d \boldsymbol{x} d s \leqslant C_{(19)}\left(|\boldsymbol{\varrho}|^{p^{-}}+h^{\frac{1}{p^{+}}}\right),
$$

with a constant $C_{(19)}>0$ independent of $\varrho=\left(\varrho_{1}, \ldots, \varrho_{d}\right)$ and $h$.

In (19) and further, we denote $\Omega_{\varrho}:=\{\boldsymbol{x} \in \Omega: \operatorname{dist}(\boldsymbol{x}, \partial \Omega)>|\varrho|\}$.

\section{Proof.}

Continuity with respect to $s$.

Set $\zeta(\boldsymbol{x}, t)=\zeta_{k}(\boldsymbol{x}, t ; s)=\left(u_{\varepsilon}(\boldsymbol{x}, s+h)-u_{\varepsilon}(\boldsymbol{x}, s)\right) \Gamma_{k}(t ; s)$, where

$$
\Gamma_{k}(t ; s)= \begin{cases}0 & \text { for } t \leqslant s \\ k(t-s) & \text { for } s \leqslant t \leqslant s+\frac{1}{k}, \\ 1 & \text { for } s+\frac{1}{k} \leqslant t \leqslant s+h-\frac{1}{k}, \\ k(s+h-t) & \text { for } s+h-\frac{1}{k} \leqslant t \leqslant s+h, \\ 0 & \text { for } t \geqslant s+h\end{cases}
$$

$k \in \mathbb{N}$ is rather large and $s$ plays the role of a parameter.

We substitute $\zeta_{k}$ for $\zeta$ into (6), which is a legal choice of a test function.

After this, we apply the formula of integration by parts [5, Section 1.5, Lemma 1.20], pass to the limit as $k \rightarrow+\infty$, and then integrate in $s$ over $(0, T-h)$. Thus, we get

$$
\int_{0}^{T-h} \int_{\Omega}\left|u_{\varepsilon}(\boldsymbol{x}, s+h)-u_{\varepsilon}(\boldsymbol{x}, s)\right|^{2} d \boldsymbol{x} d s=
$$




$$
\begin{aligned}
& =\int_{0}^{T-h} \int_{s}^{s+h} \int_{\Omega}\left(u_{\varepsilon}(\boldsymbol{x}, s+h)-u_{\varepsilon}(\boldsymbol{x}, s)\right) \partial_{t} u_{\varepsilon}(\boldsymbol{x}, t) d \boldsymbol{x} d t d s \leqslant \\
& \leqslant \int_{0}^{T-h} \int_{s}^{s+h} \int_{\Omega}\left|\nabla_{x} u_{\varepsilon}(\boldsymbol{x}, t)\right|^{p(\boldsymbol{x})-1}\left|\nabla_{x}\left(u_{\varepsilon}(\boldsymbol{x}, s+h)-u_{\varepsilon}(\boldsymbol{x}, s)\right)\right| d \boldsymbol{x} d t d s+ \\
& +\int_{0}^{T-h} \int_{s}^{s+h} \int_{\Omega} K_{\varepsilon}^{\tau}(t)\left|\beta\left(\boldsymbol{x}, u_{\varepsilon}(\boldsymbol{x}, t)\right)\right|\left|u_{\varepsilon}(\boldsymbol{x}, s+h)-u_{\varepsilon}(\boldsymbol{x}, s)\right| d \boldsymbol{x} d t d s=: \\
& =: I_{1}+I_{2} .
\end{aligned}
$$

Applying the inequality

$$
\begin{gathered}
\int_{0}^{T-h}\left(\int_{s}^{s+h} K_{\varepsilon}^{\tau}(t) d t\right) d s=\int_{0}^{h} K_{\varepsilon}^{\tau}(t) t d t+\int_{h}^{T-h} K_{\varepsilon}^{\tau}(t) h d t+ \\
\quad+\int_{T-h}^{T} K_{\varepsilon}^{\tau}(t)(T-t) d t \leqslant h \int_{0}^{T} K_{\varepsilon}^{\tau}(t) d t=h,
\end{gathered}
$$

for $0<\tau<T, \varepsilon \ll 1$, we obtain

$$
\left|I_{2}\right| \leqslant 2 M_{(13)} \sup _{(\boldsymbol{x}, z) \in \Omega \times\left(-M_{(13)}, M_{(13)}\right)}|\beta(\boldsymbol{x}, z)||\Omega| h=: M_{(21)} h .
$$

Using the Young inequality $a b \leqslant(m-1) / m(\delta a)^{m /(m-1)}+1 / m(b / \delta)^{m}$, where $1<m<\infty, a, b \geqslant 0, \delta \in(0,1)$, we estimate $\left|I_{1}\right|$ as follows:

$$
\begin{aligned}
\left|I_{1}\right| \leqslant \int_{0}^{T-h} \int_{s}^{s+h} \int_{\Omega}\left(\delta^{\frac{p(\boldsymbol{x})}{p(\boldsymbol{x})-1}} \frac{p(\boldsymbol{x})-1}{p(\boldsymbol{x})}\left|\nabla_{x} u_{\varepsilon}(\boldsymbol{x}, t)\right|^{p(\boldsymbol{x})}+\right. \\
\left.\quad+\frac{\delta^{-p(\boldsymbol{x})}}{p(\boldsymbol{x})}\left|\nabla_{x}\left(u_{\varepsilon}(\boldsymbol{x}, s+h)-u_{\varepsilon}(\boldsymbol{x}, s)\right)\right|^{p(\boldsymbol{x})}\right) d \boldsymbol{x} d t d s \leqslant \\
\leqslant \delta^{\frac{p^{+}}{p^{+}-1}} \frac{p^{+}-1}{p^{+}} h \int_{Q_{T}}\left|\nabla_{x} u_{\varepsilon}(\boldsymbol{x}, t)\right|^{p(\boldsymbol{x})} d \boldsymbol{x} d t+ \\
\quad+\frac{h}{\delta^{p^{+}}} \int_{0}^{T-h} \int_{\Omega}\left|\nabla_{x}\left(u_{\varepsilon}(\boldsymbol{x}, s+h)-u_{\varepsilon}(\boldsymbol{x}, s)\right)\right|^{p(\boldsymbol{x})} d \boldsymbol{x} d s .
\end{aligned}
$$


Choosing $\delta=h^{\left(p^{+}-1\right) /\left(p^{+}\right)^{2}}$ and taking into account (12), we get

$$
\left|I_{1}\right| \leqslant h^{\frac{1}{p^{+}}} M_{(22)}\left(T, M_{(13)}\right) \text {. }
$$

Therefore, (20), (21), and (22) give

$$
\int_{0}^{T-h} \int_{\Omega}\left(u_{\varepsilon}(\boldsymbol{x}, s+h)-u_{\varepsilon}(\boldsymbol{x}, s)\right)^{2} d \boldsymbol{x} d s \leqslant h^{\frac{1}{p^{+}}} M_{(23)} .
$$

Continuity with respect to $\boldsymbol{x}$. Using (12), we write

$$
\begin{array}{r}
\int_{0}^{T} \int_{\Omega_{\varrho}}\left|u_{\varepsilon}(\boldsymbol{x}+\boldsymbol{\varrho}, s)-u_{\varepsilon}(\boldsymbol{x}, s)\right|^{p^{-}} d \boldsymbol{x} d s \leqslant|\boldsymbol{\varrho}|^{p^{-}} C_{(24)}\left\|\nabla_{x} u_{\varepsilon}\right\|_{L^{p^{-}}\left(Q_{T}\right)}^{p^{-}} \\
\leqslant|\boldsymbol{\varrho}|^{p^{-}} M_{(24)} .
\end{array}
$$

Combining (23) and (24) and taking into account (13), we finally arrive at the inequality (19), which completes the proof of Lemma 1.

Now, from [9] we recall the following version of the Kolmogorov-Riesz theorem.

The Kolmogorov-Riesz theorem [9, Theorem 5]. Let $q \in[1,+\infty)$. A subset $\mathcal{F}$ of $L^{q}\left(\mathbb{R}^{n}\right)$ is relatively compact if, and only if,

(i) $\mathcal{F}$ is bounded in $L^{q}\left(\mathbb{R}^{n}\right)$,

(ii) for every $\epsilon>0$, there is some $R>0$, such that

$$
\int_{|\boldsymbol{y}|>R}|f(\boldsymbol{y})|^{q} d \boldsymbol{y}<\epsilon^{q}, \quad \forall f \in \mathcal{F},
$$

(iii) for every $\epsilon>0$, there is some $\delta>0$, such that

$$
\int_{\mathbb{R}^{n}}|f(\boldsymbol{y}+\overline{\boldsymbol{y}})-f(\boldsymbol{y})|^{q} d \boldsymbol{y}<\epsilon^{q}, \quad \forall f \in \mathcal{F}, \quad \forall \overline{\boldsymbol{y}} \in \mathbb{R}^{n} \text { with }|\overline{\boldsymbol{y}}|<\delta .
$$

Theorem 1, Lemma 1, and the Kolmogorov-Riesz theorem imply:

$\left\{u_{\varepsilon}\right\}_{\varepsilon>0}$ is relatively compact in $L^{q}\left(Q_{T}\right)$ for every $q \in[1,+\infty)$. 
Indeed, according to (13) and (19), the sequence $\left\{u_{\varepsilon}\right\}_{\varepsilon>0}$ is uniformly bounded and equi-continuous. Therefore, according to the KolmogorovRiesz theorem, this sequence is relatively compact in $L^{q}\left(Q_{T}\right)$, where $q \geqslant \max \left\{2, p^{-}\right\}$. Since the sequence $\left\{u_{\varepsilon}\right\}_{\varepsilon>0}$ is uniformly bounded in $L^{\infty}\left(Q_{T}\right)$, it is relatively compact in $L^{q}\left(Q_{T}\right)$ for any $q \in[1,+\infty)$.

Remark 2. The relative compactness property (25) and Theorem 1 yield that assertion 1 of Theorem 2 holds true.

4.2. Rescaling and shift. Let us fulfill some preliminary considerations before we pass to limit as $\varepsilon \rightarrow 0+$. We write out equality (6) in the unfolded form:

$$
\begin{aligned}
& \left\langle\partial_{t} u_{\varepsilon}, \zeta\right\rangle_{\mathbf{W}^{\prime}(\Omega \times(0, \tau-\varepsilon)), \mathbf{W}(\Omega \times(0, \tau-\varepsilon))}+\int_{0}^{\tau-\varepsilon} \int_{\Omega}\left|\nabla_{x} u_{\varepsilon}\right|^{p(\boldsymbol{x})-2} \nabla_{x} u_{\varepsilon} \cdot \nabla_{x} \zeta d \boldsymbol{x} d t+ \\
& \quad+\left\langle\partial_{t} u_{\varepsilon}, \zeta\right\rangle_{\mathbf{W}^{\prime}(\Omega \times(\tau-\varepsilon, \tau)), \mathbf{W}(\Omega \times(\tau-\varepsilon, \tau))}+ \\
& \quad+\int_{\tau-\varepsilon} \int_{\Omega}\left(\left|\nabla_{x} u_{\varepsilon}\right|^{p(\boldsymbol{x})-2} \nabla_{x} u_{\varepsilon} \cdot \nabla_{x} \zeta-\frac{2}{\varepsilon} K\left(\frac{t-\tau}{\varepsilon}\right) \beta\left(\boldsymbol{x}, u_{\varepsilon}\right) \zeta\right) d \boldsymbol{x} d t+ \\
& +\left\langle\partial_{t} u_{\varepsilon}, \zeta\right\rangle_{\mathbf{W}^{\prime}(\Omega \times(\tau, T)), \mathbf{W}(\Omega \times(\tau, T))}+ \\
& \quad+\int_{\tau}^{T} \int_{\Omega}\left|\nabla_{x} u_{\varepsilon}\right|^{p(\boldsymbol{x})-2} \nabla_{x} u_{\varepsilon} \cdot \nabla_{x} \zeta d \boldsymbol{x} d t=0 .
\end{aligned}
$$

We change the independent variable $t$ and the sought variable $u_{\varepsilon}$ on the segments $\{0<t<\tau-\varepsilon\}$ and $\{\tau-\varepsilon \leqslant t \leqslant \tau\}$ as follows:

For $t \in(0, \tau-\varepsilon)$, we take $\tilde{t}:=t+\varepsilon, \tilde{u}_{\varepsilon}(\boldsymbol{x}, \tilde{t}):=u_{\varepsilon}(\boldsymbol{x}, t) \equiv u_{\varepsilon}(\boldsymbol{x}, \tilde{t}-\varepsilon)$. Note that $\tilde{t} \in(\varepsilon, \tau), d t=d \tilde{t}, \partial_{t}=\partial_{\tilde{t}}$, and $t=\tilde{t}-\varepsilon$.

Following the idea of rescaling from [17], we take $\bar{t}:=\frac{t-\tau}{\varepsilon}$, $\bar{u}_{\varepsilon}(\boldsymbol{x}, \bar{t}):=u_{\varepsilon}(\boldsymbol{x}, t) \equiv u_{\varepsilon}(\boldsymbol{x}, \tau+\varepsilon \bar{t})$ for $t \in[\tau-\varepsilon, \tau]$. Note that $\tilde{t} \in[-1,0]$, $d t=\varepsilon d \bar{t}, \partial_{t}=\frac{1}{\varepsilon} \partial_{\bar{t}}$, and $t=\tau+\varepsilon \bar{t}$.

Thus, (26) takes the form

$$
\begin{aligned}
& \left\langle\partial_{\tilde{t}} \tilde{u}_{\varepsilon}(\boldsymbol{x}, \tilde{t}), \zeta(\boldsymbol{x}, \tilde{t}-\varepsilon)\right\rangle_{\mathbf{W}^{\prime}(\Omega \times(\varepsilon, \tau)), \mathbf{W}(\Omega \times(\varepsilon, \tau))}+ \\
& \quad+\int_{\varepsilon}^{\tau} \int_{\Omega}\left|\nabla_{x} \tilde{u}_{\varepsilon}(\boldsymbol{x}, \tilde{t})\right|^{p(\boldsymbol{x})-2} \nabla_{x} \tilde{u}_{\varepsilon}(\boldsymbol{x}, \tilde{t}) \cdot \nabla_{x} \zeta(\boldsymbol{x}, \tilde{t}-\varepsilon) d \boldsymbol{x} d \tilde{t}+
\end{aligned}
$$




$$
\begin{aligned}
& +\left\langle\partial_{\bar{t}} \bar{u}_{\varepsilon}(\boldsymbol{x}, \bar{t}), \zeta(\boldsymbol{x}, \tau+\varepsilon \bar{t})\right\rangle_{\mathbf{W}^{\prime}(\Omega \times(-1,0)), \mathbf{W}(\Omega \times(-1,0))}+ \\
& +\int_{-1}^{0} \int_{\Omega}\left(\varepsilon\left|\nabla_{x} \bar{u}_{\varepsilon}(\boldsymbol{x}, \bar{t})\right|^{p(\boldsymbol{x})-2} \nabla_{x} \bar{u}_{\varepsilon}(\boldsymbol{x}, \bar{t}) \cdot \nabla_{x} \zeta(\boldsymbol{x}, \tau+\varepsilon \bar{t})-\right. \\
& \left.\quad-2 K(\bar{t}) \beta\left(\boldsymbol{x}, \bar{u}_{\varepsilon}(\boldsymbol{x}, \bar{t})\right) \zeta(\boldsymbol{x}, \tau+\varepsilon \bar{t})\right) d \boldsymbol{x} d \bar{t}+ \\
& +\left\langle\partial_{t} u_{\varepsilon}(\boldsymbol{x}, t), \zeta(\boldsymbol{x}, t)\right\rangle_{\mathbf{W}^{\prime}(\Omega \times(\tau, T)), \mathbf{W}(\Omega \times(\tau, T))}+ \\
& +\int_{\tau}^{T} \int_{\Omega}\left|\nabla_{x} u_{\varepsilon}(\boldsymbol{x}, t)\right|^{p(\boldsymbol{x})-2} \nabla_{x} u_{\varepsilon}(\boldsymbol{x}, t) \cdot \nabla_{x} \zeta(\boldsymbol{x}, t) d \boldsymbol{x} d t=0 .
\end{aligned}
$$

We apply the formula of integration by parts [5, Section 1.5, Lemma $1.20]$ in $\tilde{t}, \bar{t}$ and $t$ in the respective integrals and rewrite (27) in the equivalent form:

$$
\begin{aligned}
& \int_{\Omega}\left(\tilde{u}_{\varepsilon}(\boldsymbol{x}, \tau-0) \zeta(\boldsymbol{x}, \tau-\varepsilon)-u_{0}(\boldsymbol{x}) \zeta(\boldsymbol{x}, 0)\right) d \boldsymbol{x}+ \\
& +\int_{\varepsilon} \int_{\Omega}\left(-\tilde{u}_{\varepsilon}(\boldsymbol{x}, \tilde{t}) \partial_{\tilde{t}} \zeta(\boldsymbol{x}, \tilde{t}-\varepsilon)+\right. \\
& \left.\quad+\left|\nabla_{x} \tilde{u}_{\varepsilon}(\boldsymbol{x}, \tilde{t})\right|^{p(\boldsymbol{x})-2} \nabla_{x} \tilde{u}_{\varepsilon}(\boldsymbol{x}, \tilde{t}) \cdot \nabla_{x} \zeta(\boldsymbol{x}, \tilde{t}-\varepsilon)\right) d \boldsymbol{x} d \tilde{t}+ \\
& +\int_{\Omega}\left(\bar{u}_{\varepsilon}(\boldsymbol{x}, 0-) \zeta(\boldsymbol{x}, \tau)-\bar{u}_{\varepsilon}(\boldsymbol{x},-1+0) \zeta(\boldsymbol{x}, \tau-\varepsilon)\right) d \boldsymbol{x}+ \\
& +\int_{-1}^{0} \int_{\Omega}\left(-\bar{u}_{\varepsilon}(\boldsymbol{x}, \bar{t}) \partial_{\bar{t}} \zeta(\boldsymbol{x}, \tau+\varepsilon \bar{t})-2 K(\bar{t}) \beta\left(\boldsymbol{x}, \bar{u}_{\varepsilon}(\boldsymbol{x}, \bar{t})\right) \zeta(\boldsymbol{x}, \tau+\varepsilon \bar{t})+\right. \\
& \left.\quad+\varepsilon\left|\nabla_{x} \bar{u}_{\varepsilon}(\boldsymbol{x}, \bar{t})\right|^{p(\boldsymbol{x})-2} \nabla_{x} \bar{u}_{\varepsilon}(\boldsymbol{x}, \bar{t}) \cdot \nabla_{x} \zeta(\boldsymbol{x}, \tau+\varepsilon \bar{t})\right) d \boldsymbol{x} d \bar{t}+ \\
& +\int_{\Omega}\left(u_{\varepsilon}(\boldsymbol{x}, T) \zeta(\boldsymbol{x}, T)-u_{\varepsilon}(\boldsymbol{x}, \tau+0) \zeta(\boldsymbol{x}, \tau)\right) d \boldsymbol{x}+ \\
& +\int_{\tau}^{T} \int_{\Omega}\left(-u_{\varepsilon}(\boldsymbol{x}, t) \partial_{t} \zeta(\boldsymbol{x}, t)+\right. \\
& \left.\quad+\left|\nabla_{x} u_{\varepsilon}(\boldsymbol{x}, t)\right|^{p(\boldsymbol{x})-2} \nabla_{x} u_{\varepsilon}(\boldsymbol{x}, t) \cdot \nabla_{x} \zeta(\boldsymbol{x}, t)\right) d \boldsymbol{x} d t=0 .
\end{aligned}
$$


In $(28)$ we notice that $\tilde{u}_{\varepsilon}(\boldsymbol{x}, \varepsilon)=u_{\varepsilon}(\boldsymbol{x}, 0) \stackrel{(1)_{2}}{=} u_{0}(\boldsymbol{x})(\boldsymbol{x} \in \Omega)$.

Remark 3. Also, rigorously speaking, in (28) we assume that $\partial_{\tilde{t}} \zeta, \partial_{\bar{t}} \zeta$, and $\partial_{t} \zeta$ are integrable, which is a more restrictive requirement than $\zeta \in \mathbf{Z}$, as in Definition 1. Note that the case when $\zeta$ merely belongs to $\mathbf{Z}$ is also admissible, but at the same time we should write

$$
\left\langle\partial_{\tilde{t}} \zeta(\boldsymbol{x}, \tilde{t}-\varepsilon),-\tilde{u}_{\varepsilon}(\boldsymbol{x}, \tilde{t})\right\rangle_{\mathbf{W}^{\prime}(\Omega \times(\varepsilon, \tau)), \mathbf{W}(\Omega \times(\varepsilon, \tau))}
$$

instead of $\int_{\varepsilon}^{\tau} \int_{\Omega}\left(-\tilde{u}_{\varepsilon}(\boldsymbol{x}, \tilde{t}) \partial_{\tilde{t}} \zeta(\boldsymbol{x}, \tilde{t}-\varepsilon)\right) d \boldsymbol{x} d \tilde{t}$, and so on.

Keeping in mind the further limiting passage as $\varepsilon \rightarrow 0+$, in (28) we take the test function $\zeta$ depending of $\varepsilon$ in the following form:

$$
\zeta=\zeta_{\varepsilon}(\boldsymbol{x}, t)= \begin{cases}\tilde{\zeta}(\boldsymbol{x}, \tilde{t}) \equiv \tilde{\zeta}(\boldsymbol{x}, t+\varepsilon) & \text { for } t \in[0, \tau-\varepsilon), \\ \bar{\zeta}(\boldsymbol{x}, \bar{t}) \equiv \bar{\zeta}\left(\boldsymbol{x}, \frac{t-\tau}{\varepsilon}\right) & \text { i. e., for } \tilde{t} \in[\varepsilon, \tau) ; \\ & \text { for } t \in[\tau-\varepsilon, \tau], \\ \zeta(\boldsymbol{x}, t) & \text { i. e., for } \bar{t} \in[-1,0] ; \\ \text { for } t \in(\tau, T],\end{cases}
$$

where $\tilde{\zeta}=\tilde{\zeta}(\boldsymbol{x}, \tilde{t})$ is an arbitrary smooth test function defined on $\Omega \times[0, \tau]$, $\bar{\zeta}=\bar{\zeta}(\boldsymbol{x}, \bar{t})$ is an arbitrary smooth test function defined on $\Omega \times[-1,0]$, $\zeta=\zeta(\boldsymbol{x}, t)$ is an arbitrary smooth test function defined on $\Omega \times[\tau, T]$, and the matching conditions

$$
\tilde{\zeta}(\boldsymbol{x}, \tau-0)=\bar{\zeta}(\boldsymbol{x},-1+0), \quad \bar{\zeta}(\boldsymbol{x}, 0-)=\zeta(\boldsymbol{x}, \tau+0)
$$

hold. Inserting the test function (29) into (28), we get

$$
\begin{aligned}
& \int_{\Omega}\left(\tilde{u}_{\varepsilon}(\boldsymbol{x}, \tau-0) \tilde{\zeta}(\boldsymbol{x}, \tau-0)-u_{0}(\boldsymbol{x}) \tilde{\zeta}(\boldsymbol{x}, \varepsilon)\right) d \boldsymbol{x}+ \\
& +\int_{\varepsilon} \int_{\Omega}\left(-\tilde{u}_{\varepsilon}(\boldsymbol{x}, \tilde{t}) \partial_{\tilde{t}} \tilde{\zeta}(\boldsymbol{x}, \tilde{t})+\right. \\
& \left.\quad+\left|\nabla_{x} \tilde{u}_{\varepsilon}(\boldsymbol{x}, \tilde{t})\right|^{p(\boldsymbol{x})-2} \nabla_{x} \tilde{u}_{\varepsilon}(\boldsymbol{x}, \tilde{t}) \cdot \nabla_{x} \tilde{\zeta}(\boldsymbol{x}, \tilde{t})\right) d \boldsymbol{x} d \tilde{t}+ \\
& +\int_{\Omega}\left(\bar{u}_{\varepsilon}(\boldsymbol{x}, 0-) \bar{\zeta}(\boldsymbol{x}, 0-)-\bar{u}_{\varepsilon}(\boldsymbol{x},-1+0) \bar{\zeta}(\boldsymbol{x},-1+0)\right) d \boldsymbol{x}+
\end{aligned}
$$




$$
\begin{aligned}
& +\int_{-1}^{0} \int_{\Omega}\left(-\bar{u}_{\varepsilon}(\boldsymbol{x}, \bar{t}) \partial_{\bar{t}} \bar{\zeta}(\boldsymbol{x}, \bar{t})-2 K(\bar{t}) \beta\left(\boldsymbol{x}, \bar{u}_{\varepsilon}(\boldsymbol{x}, \bar{t})\right) \bar{\zeta}(\boldsymbol{x}, \bar{t})\right. \\
& \left.\quad+\varepsilon\left|\nabla_{x} \bar{u}_{\varepsilon}(\boldsymbol{x}, \bar{t})\right|^{p(\boldsymbol{x})-2} \nabla_{x} \bar{u}_{\varepsilon}(\boldsymbol{x}, \bar{t}) \cdot \nabla_{x} \bar{\zeta}(\boldsymbol{x}, \bar{t})\right) d \boldsymbol{x} d \bar{t}+ \\
& +\int_{\Omega}\left(u_{\varepsilon}(\boldsymbol{x}, T) \zeta(\boldsymbol{x}, T)-u_{\varepsilon}(\boldsymbol{x}, \tau+0) \zeta(\boldsymbol{x}, \tau+0)\right) d \boldsymbol{x}+ \\
& +\int_{\tau}^{T} \int_{\Omega}\left(-u_{\varepsilon}(\boldsymbol{x}, t) \partial_{t} \zeta(\boldsymbol{x}, t)+\right. \\
& \left.\quad+\left|\nabla_{x} u_{\varepsilon}(\boldsymbol{x}, t)\right|^{p(\boldsymbol{x})-2} \nabla_{x} u_{\varepsilon}(\boldsymbol{x}, t) \cdot \nabla_{x} \zeta(\boldsymbol{x}, t)\right) d \boldsymbol{x} d t=0 .
\end{aligned}
$$

The rest of the proof of Theorem 2 is based on a systematical study of this integral equality.

4.3. The equality of traces. At first, note that we have, for every fixed $\varepsilon>0$ :

$$
\begin{aligned}
& \tilde{u}_{\varepsilon} \in \mathbf{W}(\Omega \times(\varepsilon, \tau)), \bar{u}_{\varepsilon} \in \mathbf{W}(\Omega \times(-1,0)), u_{\varepsilon} \in \mathbf{W}(\Omega \times(\tau, T)), \\
& \partial_{\tilde{t}} \tilde{u}_{\varepsilon} \in \mathbf{W}^{\prime}(\Omega \times(\varepsilon, \tau)), \partial_{\bar{t}} \bar{u}_{\varepsilon} \in \mathbf{W}^{\prime}(\Omega \times(-1,0)), \partial_{t} u_{\varepsilon} \in \mathbf{W}^{\prime}(\Omega \times(\tau, T))
\end{aligned}
$$

since $u_{\varepsilon} \in \mathbf{W}\left(Q_{T}\right)$ and $\partial_{t} u_{\varepsilon} \in \mathbf{W}^{\prime}\left(Q_{T}\right)$, due to Theorem 1 .

Therefore, $\tilde{u}_{\varepsilon} \in C\left([\varepsilon, \tau] ; L^{2}(\Omega)\right), \quad \bar{u}_{\varepsilon} \in C\left([-1,0] ; L^{2}(\Omega)\right)$, and $u_{\varepsilon} \in C\left([\tau, T] ; L^{2}(\Omega)\right)$ due to the well-known embedding theorem [16, Ch. 3 , Lemma 1.2]. Hence, there exist the strong (in $L^{2}(\Omega)$ ) traces $\tilde{u}_{\varepsilon}(\cdot, \tau-0)$, $\bar{u}_{\varepsilon}(\cdot,-1+0), \bar{u}_{\varepsilon}(\cdot, 0-)$, and $u_{\varepsilon}(\cdot, \tau+0)$ in $\Omega$. Moreover, the following matching property holds true for these traces:

Lemma 2. The equalities

$$
\tilde{u}_{\varepsilon}(\boldsymbol{x}, \tau-0)=\bar{u}_{\varepsilon}(\boldsymbol{x},-1+0), \quad \bar{u}_{\varepsilon}(\boldsymbol{x}, 0-)=u_{\varepsilon}(\boldsymbol{x}, \tau+0)
$$

are valid for a.e. $\boldsymbol{x} \in \Omega$ for every $\varepsilon>0$.

Proof. Due to Remark 1, we have $u_{\varepsilon}(\boldsymbol{x}, \tau-\varepsilon-0)=u_{\varepsilon}(\boldsymbol{x}, \tau-\varepsilon+0)$, which is equivalent to $(31)_{1}$, and $u_{\varepsilon}(\boldsymbol{x}, \tau-0)=u_{\varepsilon}(\boldsymbol{x}, \tau+0)$, which is equivalent to $(31)_{2}$. Lemma 2 is proved.

4.4. Passing to limit in $\Omega \times\{\boldsymbol{\tau}<\mathbf{t}<\mathbf{T}\}$. In (30), let us take $\tilde{\zeta} \equiv 0, \bar{\zeta} \equiv 0$ and an arbitrary $\zeta=\zeta(\boldsymbol{x}, t)$, such that $\zeta$ is smooth and supported in $(\tau, T)$. Thus, we get the integral equality 


$$
\begin{aligned}
& \int_{\tau}^{T} \int_{\Omega}\left(-u_{\varepsilon}(\boldsymbol{x}, t) \partial_{t} \zeta(\boldsymbol{x}, t)+\right. \\
& \left.\quad+\left|\nabla_{x} u_{\varepsilon}(\boldsymbol{x}, t)\right|^{p(\boldsymbol{x})-2} \nabla_{x} u_{\varepsilon}(\boldsymbol{x}, t) \cdot \nabla_{x} \zeta(\boldsymbol{x}, t)\right) d \boldsymbol{x} d t=0 .
\end{aligned}
$$

Due to Theorem 1 and the relative compactness property (25), we pass to the limit in (32) as $\varepsilon \rightarrow 0+$, using monotonicity of the operator $\boldsymbol{\xi} \mapsto|\boldsymbol{\xi}|^{p(\boldsymbol{x})-2} \boldsymbol{\xi}$ as in [5, Section 4.2, Lemma 4.6] and choosing a proper subsequence from $\left\{u_{\varepsilon}\right\}_{\varepsilon>0}$, if necessary; and thus we arrive at the integral equality

$$
\int_{\tau}^{T} \int_{\Omega}\left(-u(\boldsymbol{x}, t) \partial_{t} \zeta(\boldsymbol{x}, t)+\left|\nabla_{x} u(\boldsymbol{x}, t)\right|^{p(\boldsymbol{x})-2} \nabla_{x} u(\boldsymbol{x}, t) \cdot \nabla_{x} \zeta(\boldsymbol{x}, t)\right) d \boldsymbol{x} d t=0 .
$$

4.5. Passing to limit in $\boldsymbol{\Omega} \times\{\mathbf{0}<\tilde{\mathbf{t}}<\boldsymbol{\tau}\}$. Now take $\zeta \equiv 0, \bar{\zeta} \equiv 0$ and an arbitrary smooth $\tilde{\zeta}=\tilde{\zeta}(\boldsymbol{x}, \tilde{t})$ vanishing on the section $\{\tilde{t}=\tau\}$ in (30). Thus, we get the integral equality

$$
\begin{gathered}
-\int_{\Omega} u_{0}(\boldsymbol{x}) \tilde{\zeta}(\boldsymbol{x}, \varepsilon) d \boldsymbol{x}+\int_{0}^{\tau} \int_{\Omega} \mathbf{1}_{(\varepsilon<\tilde{t}<\tau)}\left(-\tilde{u}_{\varepsilon}(\boldsymbol{x}, \tilde{t}) \partial_{\tilde{t}} \tilde{\zeta}(\boldsymbol{x}, \tilde{t})+\right. \\
\left.\quad+\left|\nabla_{x} \tilde{u}_{\varepsilon}(\boldsymbol{x}, \tilde{t})\right|^{p(\boldsymbol{x})-2} \nabla_{x} \tilde{u}_{\varepsilon}(\boldsymbol{x}, \tilde{t}) \cdot \nabla_{x} \tilde{\zeta}(\boldsymbol{x}, \tilde{t})\right) d \boldsymbol{x} d \tilde{t}=0 .
\end{gathered}
$$

We pass to the limit in this integral equality as $\varepsilon \rightarrow 0+$, using the same arguments as in Section 4.4. Thus, we get

$$
\begin{aligned}
& -\int_{\Omega} u_{0}(\boldsymbol{x}) \tilde{\zeta}(\boldsymbol{x}, 0) d \boldsymbol{x}+\int_{0}^{\tau} \int_{\Omega}\left(-\tilde{u}(\boldsymbol{x}, \tilde{t}) \partial_{\tilde{t}} \tilde{\zeta}(\boldsymbol{x}, \tilde{t})+\right. \\
& \left.\quad+\left|\nabla_{x} \tilde{u}(\boldsymbol{x}, \tilde{t})\right|^{p(\boldsymbol{x})-2} \nabla_{x} \tilde{u}(\boldsymbol{x}, \tilde{t}) \cdot \nabla_{x} \tilde{\zeta}(\boldsymbol{x}, \tilde{t})\right) d \boldsymbol{x} d \tilde{t}=0,
\end{aligned}
$$

choosing a proper subsequence from $\left\{\tilde{u}_{\varepsilon}\right\}_{\varepsilon>0}$, if necessary.

Furthermore, since $\tilde{u}_{\varepsilon}(\boldsymbol{x}, \tilde{t})=u_{\varepsilon}(\boldsymbol{x}, \tilde{t}-\varepsilon)$ (for $t \in(0, \tau-\varepsilon)$ ) and the equi-continuity properties (23) and (19) hold, we deduce that

$$
\tilde{u}(\boldsymbol{x}, \tilde{t})=u(\boldsymbol{x}, \tilde{t}) \text { for a.e. }(\boldsymbol{x}, \tilde{t}) \in \Omega \times(0, \tau),
$$

where $u=u(\boldsymbol{x}, t)$ is the strong limit of the subsequence $\left\{u_{\varepsilon}\right\}_{\varepsilon \rightarrow 0+}$, which exists due to the relative compactness property (25). 
Thus, redenoting additionally $\tilde{t}:=t$, we simply write out (34) in the form

$$
\begin{aligned}
& -\int_{\Omega} u_{0}(\boldsymbol{x}) \tilde{\zeta}(\boldsymbol{x}, 0) d \boldsymbol{x}+\int_{0}^{\tau} \int_{\Omega}\left(-u(\boldsymbol{x}, t) \partial_{t} \tilde{\zeta}(\boldsymbol{x}, t)+\right. \\
& \left.\quad+\left|\nabla_{x} u(\boldsymbol{x}, t)\right|^{p(\boldsymbol{x})-2} \nabla_{x} u(\boldsymbol{x}, t) \cdot \nabla_{x} \tilde{\zeta}(\boldsymbol{x}, t)\right) d \boldsymbol{x} d t=0 .
\end{aligned}
$$

Since $u=u(\boldsymbol{x}, t)$ is the limiting point of $\left\{u_{\varepsilon}\right\}_{\varepsilon>0}$, we have

$$
u \in \mathbf{W}(\Omega \times(0, \tau)), \quad \partial_{t} u \in \mathbf{W}^{\prime}(\Omega \times(0, \tau)) .
$$

Therefore,

$$
u \in C\left([0, \tau] ; L^{2}(\Omega)\right) .
$$

The latter means, in particular, that there exist strong traces $u(\cdot, 0+)$ and $u(\cdot, \tau-0)$. By a standard procedure (see, for example, [16, Ch. 3, Sec. 1.3]) we deduce that $u(\boldsymbol{x}, 0)=u_{0}(\boldsymbol{x})$.

Besides, since $\operatorname{supp} K_{\varepsilon}^{\tau} \subset\{\tau-\varepsilon \leqslant t \leqslant \tau\}$, we see that $u_{\varepsilon}(\boldsymbol{x}, t)=u(\boldsymbol{x}, t)$ for $t \leqslant \tau-\varepsilon$. From this and (36) it follows that

$$
\left\|\tilde{u}_{\varepsilon}(\cdot, \tau-0)-\tilde{u}(\cdot, \tau-0)\right\|_{L^{2}(\Omega)} \equiv\|u(\cdot, \tau-\varepsilon)-u(\cdot, \tau-0)\|_{L^{2}(\Omega)} \underset{\varepsilon \rightarrow 0+}{\longrightarrow} 0,
$$

which means that

$$
\tilde{u}_{\varepsilon}(\cdot, \tau-0) \underset{\varepsilon \rightarrow 0+}{\longrightarrow} u(\cdot, \tau-0) \text { strongly in } L^{2}(\Omega) .
$$

4.6. Passing to limit in $\Omega \times\{-1<\overline{\mathrm{t}}<0\}$. The shock layer equation. At first, let us make the following note.

Remark 4. Relations (31) 1 and (37) imply that

$$
\bar{u}_{\varepsilon}(\cdot,-1+0) \underset{\varepsilon \rightarrow 0+}{\longrightarrow} u(\cdot, \tau-0) \text { strongly in } L^{2}(\Omega) .
$$

Further, take $\tilde{\zeta} \equiv 0, \zeta \equiv 0$ and an arbitrary smooth $\bar{\zeta}=\bar{\zeta}(\boldsymbol{x}, \bar{t})$ supported inside $\Omega \times\{-1<\bar{t}<0\}$ in (30). Thus we get

$$
\begin{aligned}
& \int_{-1}^{0} \int_{\Omega}\left(-\bar{u}_{\varepsilon}(\boldsymbol{x}, \bar{t}) \partial_{\bar{t}} \bar{\zeta}(\boldsymbol{x}, \bar{t})+\varepsilon\left|\nabla_{x} \bar{u}_{\varepsilon}(\boldsymbol{x}, \bar{t})\right|^{p(\boldsymbol{x})-2} \nabla_{x} \bar{u}_{\varepsilon}(\boldsymbol{x}, \bar{t}) \cdot \nabla_{x} \bar{\zeta}(\boldsymbol{x}, \bar{t})-\right. \\
& \left.\quad-2 K(\bar{t}) \beta\left(\boldsymbol{x}, \bar{u}_{\varepsilon}(\boldsymbol{x}, \bar{t})\right) \bar{\zeta}(\boldsymbol{x}, \bar{t})\right) d \boldsymbol{x} d \bar{t}=0
\end{aligned}
$$


By the standard reasoning, we conclude that this integral equality is equivalent in the sense of distributions to the equation

$$
\partial_{\bar{t}} \bar{u}_{\varepsilon}-2 K(\bar{t}) \beta\left(\boldsymbol{x}, \bar{u}_{\varepsilon}\right)=\varepsilon \operatorname{div}_{x}\left(\left|\nabla_{x} \bar{u}_{\varepsilon}\right|^{p(\boldsymbol{x})-2} \nabla_{x} \bar{u}_{\varepsilon}\right), \quad(\boldsymbol{x}, \bar{t}) \in \Omega \times(-1,0) .
$$

This equation is supplemented by the initial condition

$$
\left.\bar{u}_{\varepsilon}\right|_{\bar{t}=-1}=\tilde{u}_{\varepsilon}(\cdot, \tau-0)
$$

(i. e., relation $\left.(31)_{1}\right)$ and the homogeneous boundary condition $\left.\bar{u}_{\varepsilon}\right|_{\partial \Omega}=0$. Due to this condition and the finiteness condition (4), we can extend $\bar{u}_{\varepsilon}$ to the whole space $\mathbb{R}_{x}^{d}$ by zero and say that $\bar{u}_{\varepsilon}$ is the solution of the Cauchy problem $(31)_{1}$ and (38) in the space-time layer $\mathbb{R}_{x}^{d} \times(-1,0)$. We identify this Cauchy problem as the parabolic approximation of the semilinear transport equation with zero flux term:

$$
\partial_{\bar{t}} \bar{u}-2 K(\bar{t}) \beta(\boldsymbol{x}, \bar{u})=0 .
$$

Due to the theory of kinetic formulations of conservation laws, the following assertions hold true:

Lemma 3. There exist a subsequence from $\left\{\bar{u}_{\varepsilon}\right\}_{\varepsilon>0}$ (still denoted by $\left.\left\{\bar{u}_{\varepsilon}\right\}_{\varepsilon>0}\right)$ and a limiting function $\bar{u} \in L^{\infty}\left(\mathbb{R}_{x}^{d} \times(-1,0)\right)$, such that

1) $\bar{u}_{\varepsilon} \underset{\varepsilon \rightarrow 0+}{\longrightarrow} \bar{u}$ strongly in $L^{2}(\Omega \times(-1,0))$,

2) $\bar{u}$ vanishes in $\left(\mathbb{R}_{x}^{d} \backslash \Omega\right) \times[-1,0]$,

3) $\bar{u}$ is an entropy solution of the Cauchy problem for equation (39) in $\Omega \times(-1,0)$ with initial data

$$
\left.\bar{u}\right|_{\bar{t}=-1}=u(\cdot, \tau-0), \quad \boldsymbol{x} \in \Omega .
$$

The notion of entropy solution to the problem (39), (40) is standard. Namely, we say that $\bar{u}=\bar{u}(\boldsymbol{x}, \bar{t})$ is an entropy solution to the problem (39), (40) if it belongs to $L^{\infty}(\Omega \times(-1,0))$ and satisfies the integral entropy inequality

$$
\begin{aligned}
\int_{-1}^{0} \int_{\Omega}\left(\phi(\bar{u}) \partial_{\bar{t}} \bar{\zeta}+2 K(\bar{t}) \phi^{\prime}(\bar{u}) \beta(\boldsymbol{x}, \bar{u}) \bar{\zeta}\right) d \boldsymbol{x} d \bar{t}+ \\
+\int_{\Omega} \phi(u(\boldsymbol{x}, \tau-0)) \bar{\zeta}(\boldsymbol{x},-1) d \boldsymbol{x} \geqslant 0
\end{aligned}
$$


for all nonnegative smooth functions $\bar{\zeta}=\bar{\zeta}(\boldsymbol{x}, \bar{t})$ vanishing in the neighborhood of $\partial \Omega$ and the section $\{\bar{t}=0\}$ and for all smooth convex functions $\phi=\phi(w), w \in \mathbb{R}$.

In particular, (41) and inclusion $\bar{u} \in L^{\infty}(\Omega \times(-1,0))$ yield

$$
\partial_{\bar{t}} \bar{u} \in L^{\infty}(\Omega \times(-1,0))
$$

and $\bar{u}$ is, in fact, the strong generalized solution of the problem (39), (40).

Proof of Lemma 3 is achieved by the method of kinetic equation, following the proof in either [12, Ch. 3] or [13] (or [14], which is the closest consideration with regard to our present case), with some necessary modifications. These modifications are quite minor and, therefore, we omit the details.

4.7. Completion of the proof of Theorem 2. Assertion 1 of Theorem 2 has already been proved in Section 4.1, see Remark 2.

Due to Lemma 3, we conclude that assertion 2 of Theorem 2 holds true and that the limiting function $\bar{u}$ serves as a strong solution of equation (14b) in $\Omega \times(-1,0)$ and satisfies the matching condition $(14 \mathrm{e})_{1}$.

Integral equalities (33) and (35) imply that the limiting function $u$ serves as a weak (energy) solution of equation (14a) in $\Omega \times((0, \tau) \cup(\tau, T))$. Also, we have established in Section 4.5 that $u$ satisfies the initial condition (14d). Inclusion $u \in \mathbf{W}\left(Q_{T}\right)$ implies that the homogeneous condition (14c) holds.

In order to complete the proof of assertion 3 of Theorem 2, it remains to deduce $(14 \mathrm{e})_{2}$. Similarly to [13, proof of Lemma 11], we prove that

$$
\bar{u}_{\varepsilon}(\cdot, 0-) \underset{\varepsilon \rightarrow 0+}{\longrightarrow} \bar{u}(\cdot, 0-) \text { strongly in } W^{-s, 2}(\Omega),
$$

where $s>s_{d}=\left[\frac{d}{2}\right]+\frac{5}{2}$ and $\bar{u}(\cdot, 0-)$ is the trace of $\bar{u}$ on the section $\{\bar{t}=0\}$. This trace is understood in the strong sense, since the derivative $\partial_{\bar{t}} \bar{u}$ is bounded due to (42). In turn, relations $(31)_{2}$ and (43) yield that

$$
u_{\varepsilon}(\cdot, \tau+0) \underset{\varepsilon \rightarrow 0+}{\longrightarrow} u(\cdot, \tau+0)=\bar{u}(\cdot, 0-) \text { strongly in } W^{-s, 2}(\Omega) .
$$

Here $u(\cdot, \tau+0)$ is the trace of $u$ on the section $\{t=\tau+0\}$. This trace is understood in the strong sense as well, since $u \in C\left([\tau, T] ; L^{2}(\Omega)\right)$. Thus, the matching condition $(14 \mathrm{e})_{2}$ holds a. e. in $\Omega$; this completes the proof of assertion 3 . 
In order to prove assertion 4, i.e., the uniqueness result, we notice that problem (14) is, in fact, a sequence of three problems that should be solved successively. On the first step, the system (14a), (14c), and (14d) should be solved for the sought function $u$ in $\Omega \times(0, \tau)$. The solution of this problem exists and is unique due to the well-known theory of the homogeneous $p(\boldsymbol{x})$-Laplacian equation, see [2, Theorems 1 and 2].

On the second step, the system (14b) and (14e) $)_{1}$ provided with the initial data $\left.\bar{u}\right|_{\bar{t}=-1}=u(\boldsymbol{x}, \tau-0)$ should be solved for the sought function $\bar{t} \mapsto \bar{u}(\boldsymbol{x}, \bar{t})$ on $[-1,0]$. The variable $\boldsymbol{x} \in \Omega$ plays the role of a parameter in this formulation, and the initial function $u(\cdot, \tau-0)$ is defined on the first step. Due to the classical theory of the first-order ordinary differential equations [10, Ch. 1, Sec. 1.7, Theorems 1 and 2], this problem has a unique solution for a.e. $\boldsymbol{x} \in \Omega$, since $K$ and $\beta$ are smooth functions.

On the third (final) step, the system (14a), (14c), and (14e) $)_{2}$ provided with the initial data $\left.u\right|_{t=\tau}=\bar{u}(\boldsymbol{x}, 0-)$ should be solved for the sought function $u$ in $\Omega \times(\tau, T)$. The initial function $\bar{u}(\cdot, 0-)$ is defined on the second step. The solution of this problem exists and is unique due to the same arguments, as on the first step.

Thus, the solution of problem (14) is unique. This conclusion completes justification of Theorem 2 .

5. Concluding remarks. Due to the uniqueness assertion of Theorem 2, i. e., assertion 4 , we conclude that the whole family $\left\{u_{\varepsilon}\right\}_{\varepsilon>0}$ of solutions to problem (1) converges to the generalized solution of the system (14) as $\varepsilon \rightarrow 0+$.

Further, from the system (14) it is clear that its solution $u=\lim _{\varepsilon \rightarrow 0+} u_{\varepsilon}$ does not meet the standard impulsive condition (8). Actually, $u$ satisfies (8) only in the cases when $\beta$ is independent of $u$, i. e., $\beta(\boldsymbol{x}, u) \equiv \beta(\boldsymbol{x})$. In particular, even in the cases when $\beta$ is linear in $u$, the problem (1) as $\varepsilon \rightarrow+0$ yields another impulsive condition than (8), which resembles the simple example exposed in Introduction.

Finally, let us notice that, due to the existing theory of anisotropic parabolic equations with non-standard growth [5, Ch. 4], all arguments in this article can be naturally adapted for the study of the anisotropic $\boldsymbol{p}(\boldsymbol{x})$-Laplacian equation with the minor term collapsing as $\varepsilon \rightarrow 0+$ :

$$
\partial_{t} u_{\varepsilon}=\sum_{i=1}^{d} \partial_{x_{i}}\left(\left|\partial_{x_{i}} u_{\varepsilon}\right|^{p_{i}(\boldsymbol{x})-2} \partial_{x_{i}} u_{\varepsilon}\right)+K_{\varepsilon}^{\tau}(t) \beta\left(\boldsymbol{x}, u_{\varepsilon}\right), \quad(\boldsymbol{x}, t) \in Q_{T} .
$$

Correspondingly, the results, similar to Theorems 1 and 2, are valid for 
this equation.

Acknowledgment. The formulation and proof of Theorem 1 were supported by the Russian Scientific Foundation (RSF grant no. 19-11-00069). The writing of Section 1, the formulation and proof of Theorem 2 were supported by the FCT, Portugal Project UID/MAT/04561/2019, and by the Ministry of Science and Higher Education of the Russian Federation (project no. III.22.4.2).

\section{References}

[1] Agarwal R., Hristova S., O'Regan D. Non-Instantaneous Impulses in Differential Equations. Springer. 2017.

DOI: https://doi.org/10.1007/978-3-319-66384-5

[2] Alkhutov Y. A., Zhikov V. V. Existence theorems for solutions of parabolic equations with a variable order of nonlinearity. Tr. Mat. Inst. Steklova. 2010, vol. 270, pp. 21-32.

DOI: https://doi.org/10.1134/S0081543810030028

[3] Alkhutov Y. A., Zhikov V. V. Existence and uniqueness theorems for solutions of parabolic equations with a variable nonlinearity exponent. Sbornik: Mathematics. 2014, vol. 205, no. 3, pp. 307-318.

DOI: https://doi.org/10.1070/SM2014v205n03ABEH004377

[4] Antontsev S., Kuznetsov I., Shmarev S. Global higher regularity of solutions to singular $p(x, t)$-parabolic equations. J. Math. Anal. Appl. 2018, vol. 466, no. 1 , pp. 238-263.

DOI: https://doi.org/10.1016/j.jmaa.2018.05.075

[5] Antontsev S., Shmarev S. Evolution PDEs with Nonstandard Growth Conditions: Existence, Uniqueness, Localization, Blow-up. Atlantis Press. 2015. DOI: https://doi.org/10.2991/978-94-6239-112-3

[6] Coutinho F. A. B., Nogami Y., Toyama F. M. Unusual situations that arise with the Dirac delta function and its derivative. Revista Brasileira de Ensino de Física. 2009, vol. 31, no. 4 (4302), pp. 1-7.

DOI: https://doi.org/10.1590/S1806-11172009000400004

[7] Diening L., Nägele P., Ružička M. Monotone operator theory for unsteady problems in variable exponent spaces. Complex Var. Elliptic Equ. 2012, vol. 57, no. 11 , pp. $1209-1231$.

DOI: https://doi.org/10.1080/17476933.2011.557157

[8] Griffiths D., Walborn S. Dirac deltas and discontinuous functions. Amer. J. Phys. 1999, vol. 67, p. 446. DOI: https://doi.org/10.1119/1.19283 
[9] Hanche-Olsen H., Holden H. The Kolmogorov-Riesz compactness theorem. Expositiones Math. 2010, vol. 28, pp. 385-394.

DOI: https://doi.org/10.1016/j.exmath.2010.03.001

[10] Kreyszig E. Advanced Engineering Mathematics. 10th edition, John Wiley \& Sons Inc., 2011. DOI: https://doi.org/10.1002/bimj.19650070232

[11] Miller B. M., Rubinovich E. Ya. Impulsive Control in Continuous and Discrete-Continuous Systems. Springer, 2003.

DOI: https://doi.org/10.1007/978-1-4615-0095-7

[12] Perthame B. Kinetic Formulations of Conservation Laws. Oxford: Oxford Univ. Press, 2002.

[13] Plotnikov P. I., Sazhenkov S. A. Kinetic formulation for the Graetz-Nusselt ultra-parabolic equation. J. Math. Anal. Appl. 2005, vol. 304, pp. 703-724. DOI: https://doi.org/10.1016/j.jmaa.2004.09.050

[14] Sazhenkov S. A. Noisiness estimate for kinetic solutions of scalar conservation laws. In: Rodionov E. D. (ed.) Collection of scientific works (electronic resource), International Conference 'Lomonosov's Readings on Altai: Fundamental Problems of Science and Technology - 2018', pp. 249-257. Barnaul, Altai State University Press, 2018.

[15] Serrin J. Mathematical Principles of Classical Fluid Mechanics. In: Truesdell C. (ed.) Fluid Dynamics I. Encyclopedia of Physics, vol. 3/8/1, pp. 125-263. Springer, Berlin, Heidelberg, 1959.

DOI: https://doi.org/10.1007/978-3-642-45914-6

[16] Temam R. Navier-Stokes Equations: Theory and Numerical Analysis. (AMS Chelsea Publishing). 2000.

DOI: https://doi.org/10.1090/chel/343

[17] Vasseur A. Well-posedness of scalar conservation laws with singular sources. Methods Appl. Anal. 2002, vol. 9, no. 2, pp. 291-312.

DOI: https://doi.org/10.4310/MAA.2002.v9.n2.a6

[18] Wang J., Fečkan M. Non-Instantaneous Impulsive Differential Equations. Basic Theory and Computation, IOP Publishing, 2018.

DOI: https://doi.org/10.1088/2053-2563/aada21

Received August 25, 2020.

In revised form, October 28, 2020.

Accepted October 28, 2020.

Published online November 3, 2020. 
S. N. Antontsev ${ }^{a, b}$, I. V. Kuznetsov ${ }^{b, c}$, and S. A. Sazhenkov ${ }^{b, c}$

a. CMAF-CIO, University of Lisbon

Campo Grande 1749-016, Lisbon, Portugal

antontsevsn@mail.ru

b. Lavrentyev Institute of Hydrodynamics SB RAS

630090, 15, Prospect Lavrentyeva, Novosibirsk, Russia

kuznetsov_i@hydro.nsc.ru, sazhenkovs@yandex.ru

c. Novosibirsk State University

630090, 1, Pirogova Street, Novosibirsk , Russia 